\title{
Study on vibration characteristics of high speed train gear box
}

\author{
YuQing Yuan ${ }^{1, a^{*}}$, Qiang $\mathrm{Li}^{1, \mathrm{~b}}$, Guang Yang ${ }^{1, \mathrm{c}}$ \\ ${ }^{1}$ Engineering Research Center of Structure Reliability and Operation Measurement Technology of \\ Rail Guided Vehicles, Ministry of Education, Beijing Jiaotong University. Beijing, China \\ a10116309@bjtu.edu.cn, ${ }^{\mathrm{b}} \mathrm{q}$ li3@bjtu.edu.cn, ${ }^{\mathrm{c}} 11116340 @$ bjtu.edu.cn
}

Keywords: Gearbox, Line test, Spectrum analysis, Vibration characteristics.

Abstract. A large number of cracks were found in the gear box of high speed train in practical applications. According to the line test, the weak position was got and the reason of cracks was researched. The influence of the different vibration source on the weak part was studied by means of vibration spectrum analysis. The vibration transfer characteristics of 4 kinds of gear box are studied and the vibration transfer law was analysed. The results show that: the gearbox vibration is mainly influenced by the vibration of the axle box, the vibration of the motor and the gear meshing vibration. The low frequency vibration of the gear box is mainly from the axle box vibration, and the high frequency vibration is mainly influenced by the gear meshing vibration.

\section{Introduction}

In recent years, high speed train had a rapid development. As the key part of the high speed train, the gear box is a complicated mechanical system. The gear box is suspended on the motor axle and directly under the impact of the rail on the wheel in the normal operation. The working environment of gear box is very bad ${ }^{[1]}$. Its security and stability directly affect the operation reliability of emu. In our country, since the first case of gear box crack failure was found in 2012, up to now, there are more than 30 cases occurred in a brand of gear box. The gear box cracks have become the main failure form of high speed train. These problems are mainly caused by the fatigue and vibration, which will bring serious threat to the safety of vehicle.

Since the 70's in twentieth Century, many scholars have made a lot of efforts and contributions to the research and development of gear box vibration. Zhu Ge and Ding Kang have researched the mechanism of the gear vibration under the ideal condition. The result showed that the vibration was due to the characteristics of the gear tooth profile. And the fault form and vibration characteristics of gear were analyzed. The characteristic of the gear vibration frequency band was obtained ${ }^{[2][3]}$. Dong Hong and Wang Biqin had a fault diagnosis of abnormal vibration of gear box of rolling mill by use of spectrum analysis method based on the gears meshing mechanics model. The cause of abnormal vibration of gear box was obtained, and it was found that this is consistent with the actual situation ${ }^{[4]}$. However, there was a common place in the gearbox system, which was the gear box system was in a static environment, all the excitation came from the system itself. Therefore, in the paper, the vibration characteristics of high speed train gear box will be further studied under the operating environment based on their research.

\section{Line teat of gear box}

The test object is the gear box used by the EMU, the 4 kinds of gear boxes are named as A, B, C, and $\mathrm{D}$. The measuring points are located in the main position of each gear box system. The structure of the gear box is different, but the position of the test and the test condition are consistent. The gear box structure and the measuring point position are shown in Fig. 1. 


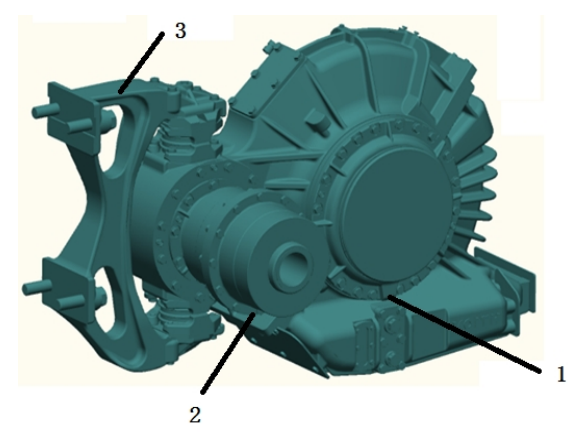

(a) Gear box A and measuring point

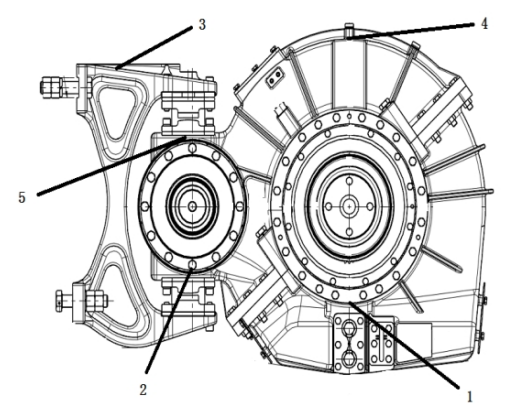

(c) Gear box C and measuring point

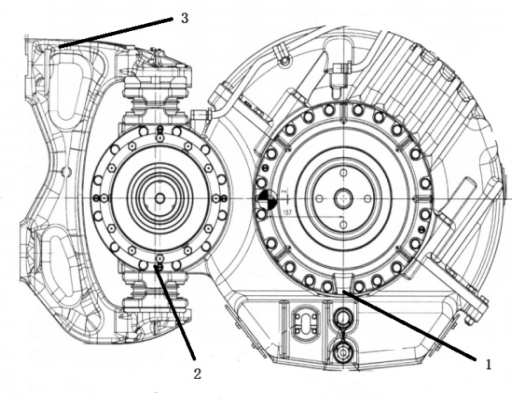

(b) Gear box B and measuring point

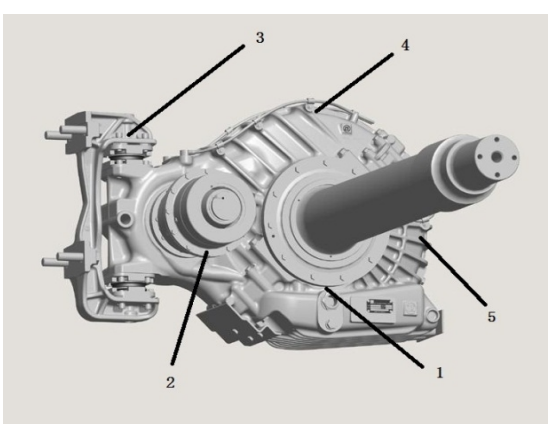

(d) Gear box D and measuring point

Fig. 1 Test gear box structure and measuring point

\section{Vibration transfer characteristics of gear box system}

There are three research methods of the transmission characteristics of the gear box system: time domain analysis, frequency domain analysis and acceleration spectrum analysis ${ }^{[5][6]}$.

Gear box A and B were located on the same train, different axle. The test condition was completely consistent. Test results are shown in Fig. 2 and Fig. 3:

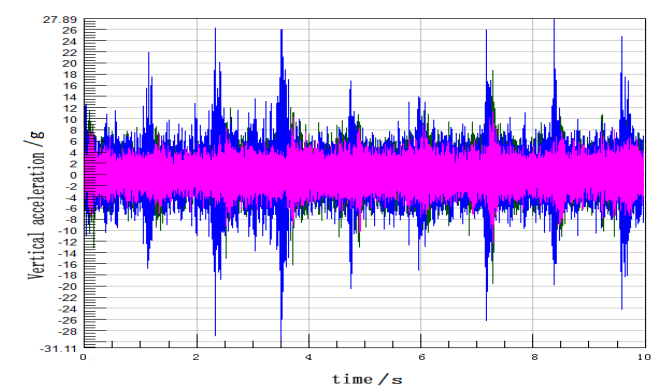

(a) Vertical acceleration time domain chart

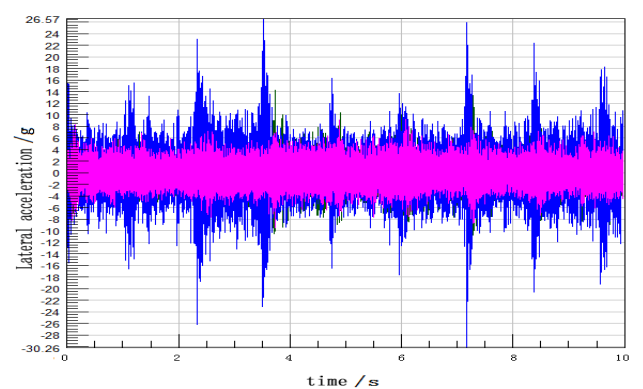

(b) Lateral acceleration time domain chart Fig. 2 Acceleration time domain chart of gear box A

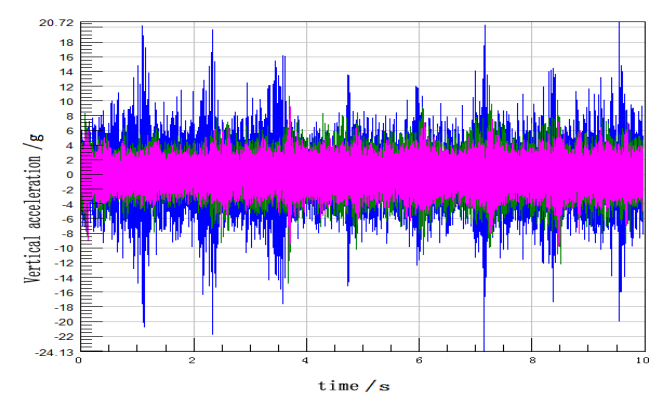

(a) Vertical acceleration time domain chart

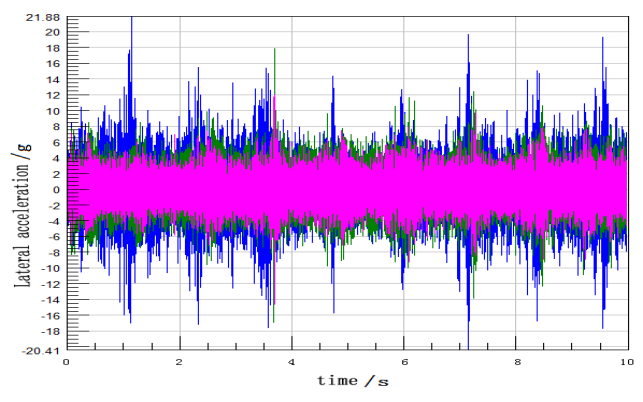

(b) Lateral acceleration time domain chart Fig. 3 Acceleration time domain chart of gear box B 
In Fig. 2 and Fig. 3, blue for the acceleration of axle box, pink for the acceleration of measuring point 1(LSS) and green for the acceleration of measuring point 2 (HSS). The acceleration vibration of the gear box $\mathrm{A}$ is consistent with the axle box acceleration. The lateral and vertical acceleration amplitude of the gear box measuring points was smaller than that of the axle box. The vibration amplitude of the measuring point 1 (LSS) is larger than that of the test point 2 (HSS), and the vibration amplitude of the measuring point 3 (The $\mathrm{C}$ type bracket) is much smaller than that of the gear box and the axle box.

The test result of gear box B is similar to gear box A, but the subtraction is more obvious at the acceleration amplitude, which indicates that suppression of vibration transfer was better. In the horizontal comparison, the amplitude of gear box B is smaller than that of the gear box A, the vertical acceleration amplitude of LSS was reduced by $14 \%$, the lateral acceleration amplitude was reduced by $12.6 \%$. The degree of attenuation is obvious

FFT transform of the time domain signal was carried out, and the frequency domain of the measuring points was obtained, as shown in Fig. 4 and Fig. 5

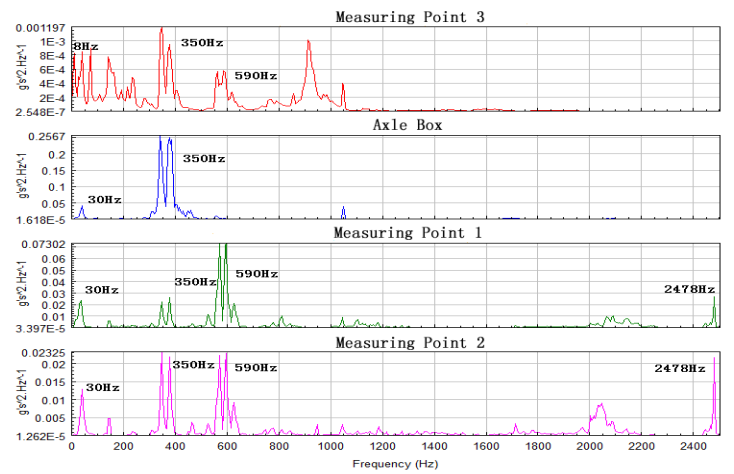

(a) the vertical acceleration

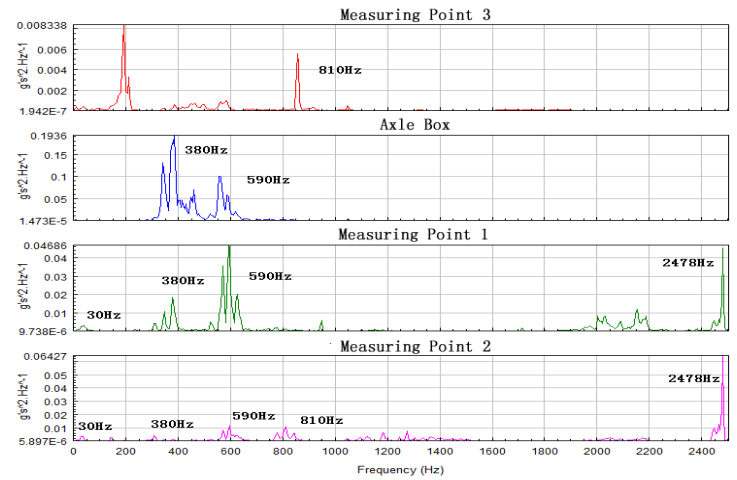

(b) the lateral acceleration

Fig. 4 acceleration frequency domain chart of gear box A

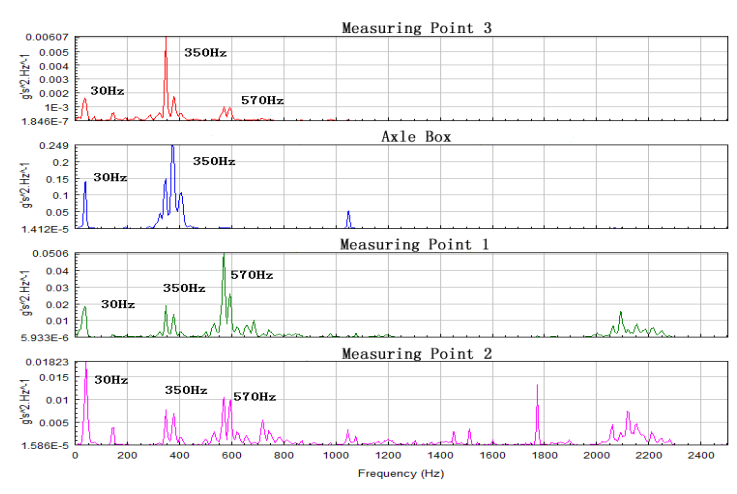

(a) the vertical acceleration

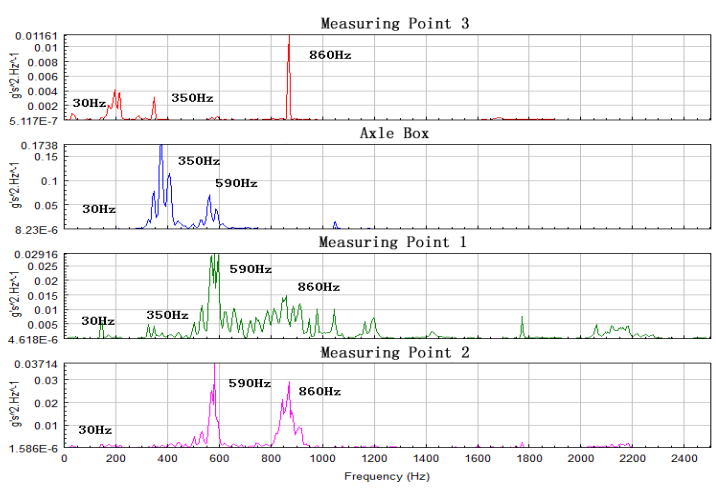

(b) the lateral acceleration

Fig. 5 acceleration frequency domain chart of gear box B

The vibration frequency distribution of the two kinds of gear box was basically the same. They were both very scattered. In the low frequency range, the vibration dominant frequency of axle box was $30 \mathrm{~Hz}$ and $350 \mathrm{~Hz}$, and there was almost no dominant frequency in the high frequency range. For the gear box, the dominant frequency was $30 \mathrm{~Hz}, 350 \mathrm{~Hz}$ and $590 \mathrm{~Hz}$, and there are some individual dominant frequencies in the range of high frequency. By vehicle speed, $30 \mathrm{~Hz}$ is the wheel rotation frequency. The frequency component of vertical acceleration is more than lateral acceleration. Besides $350 \mathrm{~Hz}$, the lateral acceleration of the axle box has the peak value of $590 \mathrm{~Hz}$.

The vertical and lateral vibration frequency energy value of the gear box B is smaller than that of the gear box A. In the high frequency range of gear box A, there was the dominant frequency about $2478 \mathrm{~Hz}$, which was the frequency of the gear mesh. It was showed that the high frequency characteristic of gear mesh is present in the actual operation, and the performance of the gear box A was obvious and the influence of the box was larger. In the vertical frequency components of gear box 
$\mathrm{B}$, the vibration energy of $30 \mathrm{~Hz}$ which caused by the wheel rotation, was higher than that of the gear box A. It was showed that the transmission of the low frequency range of the gear box B was better.

In order to research the vibration transfer law and the vibration energy variation trend of the gear box system, the concept of the acceleration spectrum was introduced. The corresponding relationship between the amplitude and frequency of the vibration was studied, and the acceleration value of the different measuring points was compared. The acceleration spectrum of gear box A and B was established, as shown in Fig. 6 and Fig. 7:

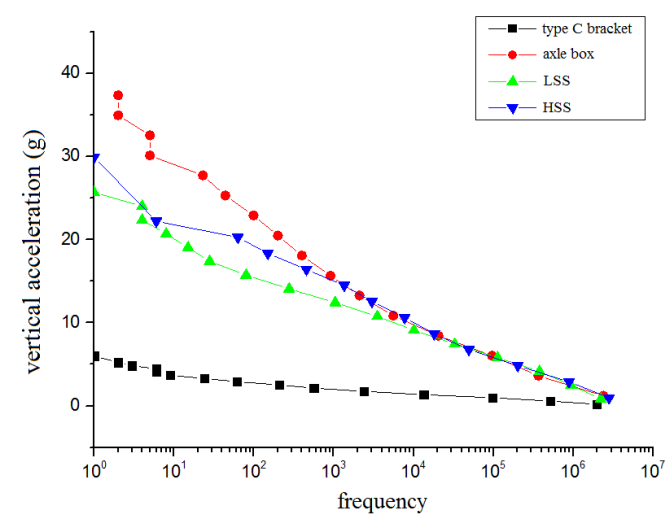

(a) The vertical acceleration discrete graph

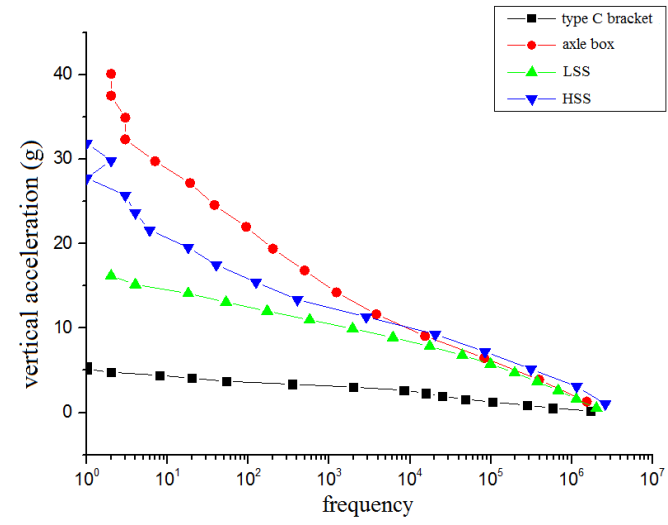

(b) The lateral acceleration discrete graph

Fig. 6 Acceleration discrete graph of gearbox A

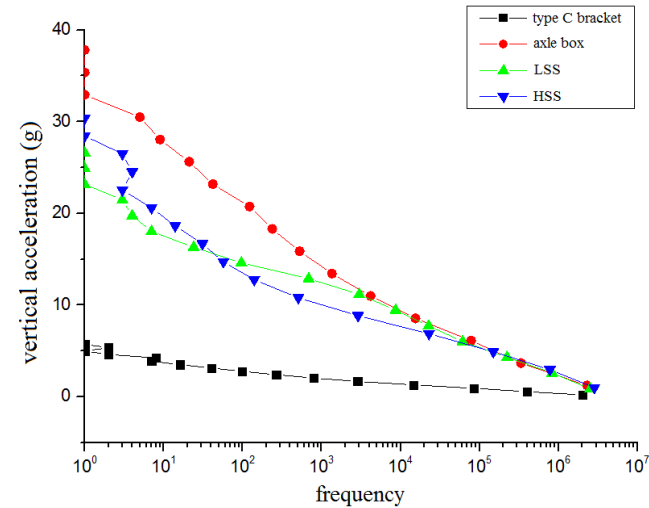

(a) The vertical acceleration discrete graph

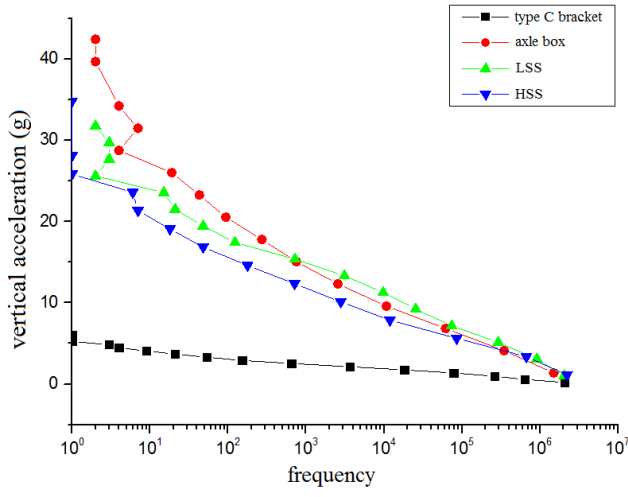

(b) The lateral acceleration discrete graph

Fig. 7 Acceleration discrete graph of gearbox B

For the gear box A, the acceleration amplitude of the axle box was larger than that of the gear box in every order. In the low frequency range, the difference value of acceleration amplitude was larger, but in high frequency range the difference value of acceleration amplitude gradually decreases, finally tends to be consistent. Further analysis was done to the acceleration amplitude attenuation law in the low frequency range, the amplitude of vertical acceleration was attenuated by $31.2 \%$ and the amplitude of lateral acceleration was attenuated by $59.6 \%$ from the axle box to the gear box. The amplitude of vertical acceleration was attenuated by $14.2 \%$ and the amplitude of lateral acceleration was attenuated by $49.2 \%$ from the HSS to the LSS.

Through the analysis of the amplitude distribution law, the cause of the vibration of LSS was the transmission from the axle box and the HSS in the gear box A. The vertical acceleration was affected more than the lateral acceleration.

Similar conclusion can be obtained by comparing the data of gear box B. In the low frequency range, the amplitude of vertical acceleration was attenuated by $29.6 \%$ and the amplitude of lateral acceleration was attenuated by $25.1 \%$ from the axle box to the gear box. The amplitude of vertical acceleration was attenuated by $12.5 \%$ and the amplitude of lateral acceleration was attenuated by $8.8 \%$ from the HSS to the LSS.

The test line and test condition of the gearbox A and B were the same. So the comparison analysis was made. Firstly, the vibration law of the same measuring position was the same: the acceleration of 
the axle box was the highest, and the LSS is the lowest. Secondly, the change trend of the acceleration amplitude was also consistent, but there was a significant difference in the degree of attenuation. For the two kinds of gear box, the vertical acceleration amplitude change trend was similarly. The attenuation of gear box B was a little smaller. For the lateral acceleration, the two kinds of gear box were obviously different. The attenuation of lateral acceleration of gear box A was significantly higher than that of B. Compared with the lateral acceleration of the gear box A and B. The LSS lateral acceleration of gear box B was increased by $131.6 \%$ compared with gear box A. The acceleration of the axle box B is increased by $5.6 \%$ compared with $\mathrm{A}$, which indicates the gear box $\mathrm{A}$ is better than the gear box $\mathrm{B}$ in the suppression of lateral vibration.

Analysis of the test results of the gear box $\mathrm{C}$ and $\mathrm{D}$ was done by the same method.

The structure of gear box $\mathrm{C}$ is similar to the gear box A except the fuel tank tail. Gear box $\mathrm{C}$ increased the measuring point of the motor and gear box upper portion. The vertical acceleration of the measuring point 3 was equivalent to the axle box, but the measuring point 4 was about 7 times higher than the box, and measuring point 1 was about 4 times than higher. The degree of the increase of the lateral acceleration amplitude was more obvious. Vibration energy mainly exists in the gear box upper portion in the High frequency range, and the oil hole in the low frequency range. The vibration energy of the axle box and $\mathrm{C}$ type bracket was small. The acceleration spectrum of gear box $\mathrm{C}$ was established, as shown in Fig. 8:

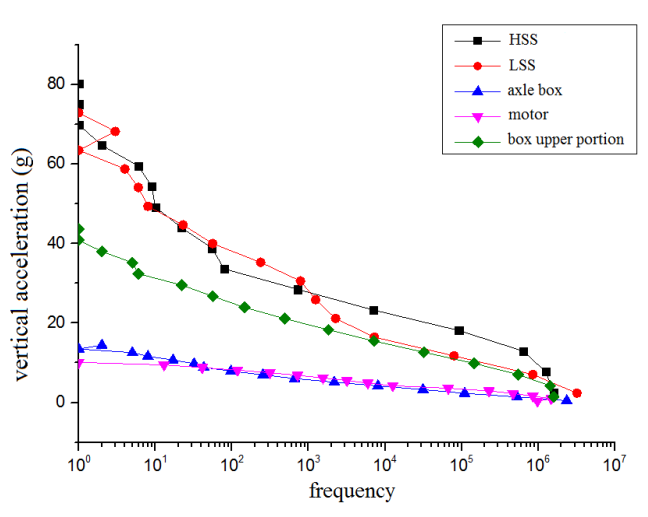

(a) The vertical acceleration discrete graph

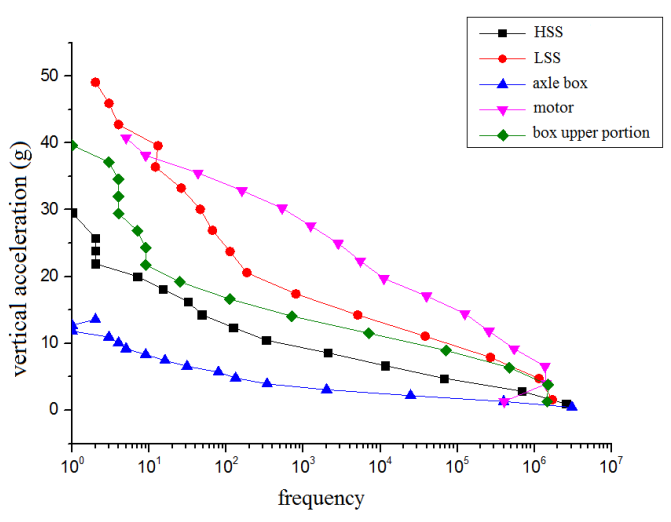

(b) The lateral acceleration discrete graph

Fig. 8 Acceleration discrete graph of gear box $\mathrm{C}$

The vertical and lateral vibration of the LSS was very violent, and the vibration of the axle box was weak. In the low frequency range, the vertical acceleration of the LSS was about 5 times higher than the axle box; HSS was about 8 times higher than the motor. The acceleration was attenuated by $45.6 \%$ from the HHS to gear box upper portion, and there were $40.1 \%$ of the attenuation from the LSS to the gear box upper portion. The lateral acceleration was attenuated by $27.4 \%$ from the motor to the HSS, and there were $19.1 \%$ of the attenuation from the LSS to the gear box upper portion

Through the analysis of above, it can be known that the vibration of the gear box is caused by the gear meshing, instead of the axle box. The vibration amplitude of the HSS and LSS is higher than that of the gear box upper portion, which indicates that the vibration is mainly located in the lower part of the gear box. The lateral vibration amplitude of the HSS is lower than that of the LSS and the gear box upper portion, and the vibration of the motor is obviously enlarged. The vibration of the HSS was mainly derived from the motor.

The structure of the gear box D is more streamlined; it can reduce the stress concentration. The acceleration spectrum of gear box D was established, as shown in Fig. 9. The acceleration amplitude-frequency relationship of gear box D was different from gear box A, B and C

The vibration of LSS was increased from the axle box; it can be known that the vibration of the gear box is caused by the gear meshing, instead of the axle box. The vibration amplitude of the HSS and LSS is higher than that of the gear box upper portion, which indicates that the vibration is mainly located in the lower part of the gear box. The vibration of Gear box tail and LSS were large. The gear box has an elongated tail structure, so transverse wagging vibration is likely to occur in the operation process. 


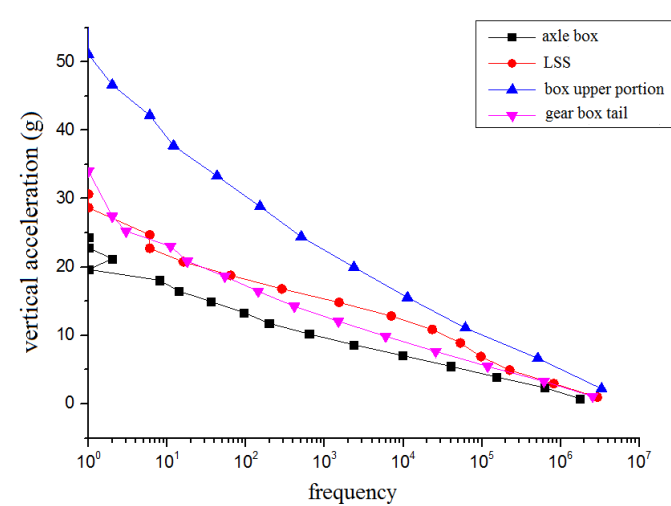

(a) The vertical acceleration discrete graph

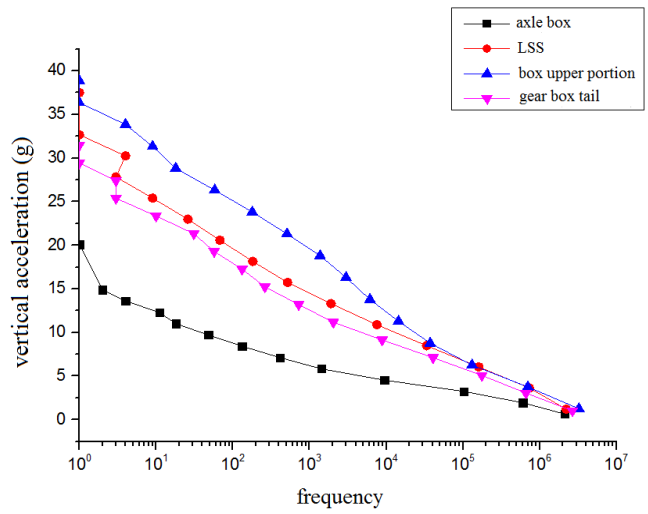

(b) The lateral acceleration discrete graph

Fig. 9 Acceleration discrete graph of gear box D

\section{Conclusions}

1) Vibration of gear box A and B are attenuated compared to the axle box, indicating that the gearbox has a good inhibition for vibration transmission. Vibration affected by the axle box in the low frequency range and the gear meshing in the high frequency range. The influence of meshing vibration on the gear box A is greater. The gear box A is better than the gear box B in the suppression of lateral vibration.

2) The vibration of the gear box $\mathrm{C}$ is caused by the gear meshing, instead of the axle box. The lateral vibration of the HSS was mainly derived from the motor. The transverse wagging vibration is likely to occur in the operation of gear box D.

3) The vibration of the gear box is mainly influenced by the vibration of the axle box, the motor and the gear meshing. Because of the different structure of the gear box, the gear box has a different degree of amplification or attenuation of the axle box vibration.

4) In the low frequency range, vibration of the gear box is influenced by the vibration of the axle box most. The high frequency vibration of gear meshing has a different influence for different structure of gear box. The vibration of the motor has a significant influence on the lateral vibration, and the vertical vibration is greatly influenced by the wheel / rail interaction.

\section{References}

[1] Zhenwei Huang. Development of the axle gear box for EMU, J. Diesel locomotives, 2009(3):14-16. (In Chinese)

[2] Kang Ding. Practical technology for fault diagnosis of gear and gear box, M. Machinery Industry Press. Beijing 2005. (In Chinese)

[3] Ge Zhu. Research on new method of gear noise demodulation analysis and the research on the subjective evaluation system of acoustic quality, D. Chong Qing, 2003.9 (In Chinese)

[4] Hong Dong, Wang Biqin. Analysis and fault diagnosis of abnormal vibration of gear box of rolling mill, J. Chinese equipment engineering 2005 (07):46-47 (In Chinese)

[5] Zunsong Ren, Zhiming Liu. Vibration transmission and frequency distribution of high speed EMU, J. Mechanical engineering. 2013, 49 (16), 1-7. (In Chinese)

[6] Lei $\mathrm{Hu}$, analysis and Research on the vibration response analysis and diagnosis technology of gear box, D. Wuhan, 2012 (In Chinese) 